\title{
Efficacy and safety of apatinib combined with whole-brain radiation therapy with a simultaneous integrated boost for brain metastases from non-small cell lung cancer: a multicenter retrospective study
}

\author{
Jia $\mathrm{Ma}^{1 \#}$, Jianping Bi ${ }^{1 \#}$, Xiulin Tuo ${ }^{2}$, Guoliang $\mathbf{P i}^{1}$, Ying $\mathrm{Li}^{1}$, Yanping $\mathrm{Li}^{1}$, Fanyu Zeng ${ }^{1}$, Hongyun Gong ${ }^{3}$, \\ Desheng $\mathrm{Hu}^{1}$, Guang $\mathrm{Han}^{1}$ \\ ${ }^{1}$ Department of Radiation Oncology, Hubei Cancer Hospital, Tongji Medical College, Huazhong University of Science and Technology, Wuhan, \\ China; ${ }^{2}$ Department of Oncology, Jianghan Oilfield General Hospital, Qianjiang, China; ${ }^{3}$ Department of Oncology, Renmin Hospital of Wuhan \\ University, Wuhan, China \\ Contributions: (I) Conception and design: D Hu, G Han; (II) Administrative support: Y Li, F Zeng; (III) Provision of study materials or patients: J Bi, \\ G Pi, Y Li; (IV) Collection and assembly of data: J Ma, J Bi; (V) Data analysis and interpretation: J Ma, J Bi; (VI) Manuscript writing: All authors; (VII) \\ Final approval of manuscript: All authors. \\ "These authors contributed equally to this work. \\ Correspondence to: Guang Han. Department of Radiation Oncology, Hubei Cancer Hospital, Tongji Medical College, Huazhong University of Science \\ and Technology, Wuhan, China. Email: hg7913@hotmail.com; Desheng Hu. Department of Radiation Oncology, Hubei Cancer Hospital, Tongji \\ Medical College, Huazhong University of Science and Technology, Wuhan, China. Email: hds_005@163.com.
}

Background: Brain metastases (BMs) develop in 20-65\% of non-small cell lung cancer (NSCLC) patients and are associated with a poor prognosis. Apatinib, a tyrosine kinase inhibitor (TKI) that selectively inhibits the vascular endothelial growth factor receptor 2, is safe and significantly prolongs the survival of chemotherapy-refractory gastric cancer patients. This retrospective study evaluated the safety and efficacy of apatinib combined with concurrent brain radiotherapy in NSCLC patients with BMs.

Methods: This trial enrolled patients with non-recurrent BM from histologically-confirmed NSCLC without any limits regarding the BM size/quantity. Eligibility criteria were patients 18-75 years old with measurable BM from histologically-confirmed NSCLC (including both newly-diagnosed and previously treated NSCLC) and expected survival time greater than 3 months. Oral apatinib (500 or $250 \mathrm{mg} /$ day) was started within 1 week prior to commencing whole brain radiotherapy with simultaneous integrated boost (WBRT-SIB) and continued until one week after radiotherapy completion. In addition to toxicities, analyzed outcomes included intracranial overall response rate (iORR), intracranial disease control rate (iDCR), intracranial progression free survival (iPFS), and overall survival (OS).

Results: From July 2016 to January 2020, 16 patients were enrolled in this retrospective study. After 3 months of brain radiotherapy, the iORR was $75 \%$, the iDCR was $100 \%$, and the brain edema index (EI) was significantly reduced compared to that before brain radiation therapy (4.2 vs. 1.9; $\mathrm{P}=0.02$ ). The median iPFS was 16.5 months [95\% confidence interval (CI): 15.1-37.4 months]. The median OS was 26 months (95\% CI: 17.0-54.0 months). Most of the patients tolerated apatinib well, but 7 patients had side effects, most commonly grade 1 or 2 . Only 2 patients experienced grade 3 adverse events (hypertension and oral mucositis), and no grade 4 or 5 toxicities were observed.

Conclusions: Apatinib combined with WBRT-SIB appears to be safe and effective in treating BMs in NSCLC patients.

Keywords: Non-small cell lung cancer (NSCLC); brain metastases (BMs); apatinib; brain radiotherapy

Submitted Dec 29, 2021. Accepted for publication Feb 22, 2022.

doi: 10.21037/jtd-22-96

View this article at: https://dx.doi.org/10.21037/jtd-22-96 


\section{Introduction}

The brain is a common metastatic site of lung cancer. Approximately $10-20 \%$ of lung cancer patients have brain metastases (BMs) at initial diagnosis (1). Further, about $20-65 \%$ of non-small cell lung cancer (NSCLC) patients will develop BMs during treatment (2). With the emergence of new treatment methods, such as targeted therapy and immunotherapy, the survival time of lung cancer is prolonged, but the probability of BM also increases (3). The occurrence of BMs often leads to severe headaches, nausea, vomiting, and neurological disorders, such as a slow response, dementia, and seizures $(4,5)$. Additionally, patients' quality of life is significantly reduced, and the natural average survival time is $<3-6$ months (6). Thus, the treatment of BMs in lung cancer patients is a very common and thorny problem in clinical setting.

Angiogenesis is a critical step in the occurrence and development of cancer. The vascular endothelial growth factor (VEGF) and its receptor vascular endothelial growth factor receptor (VEGFR) are critical in anti-angiogenic targeted therapy $(7,8)$. Several clinical studies such as ECOG 4599, and ALTER 0303/1202 confirmed the benefits of antiangionenic drugs in lung cancer, which prolongs progression free survival (PFS) and overall survival (OS). compared to chemotherapy alone (9-11). Therefore, Avastin and anlotinib were approved in first-line and third-line treatment for advanced non-squamous non-small cell lung cancer and lung cancer respectively. Apatinib is a small-molecule tyrosine kinase inhibitor (TKI) that specifically inhibits the VEGFR-2, and is used in the standard treatment of advanced gastric cancer $(12,13)$. This drug is also used to treat a variety of solid tumors, and can prolong OS (14).

Whole-brain radiation therapy (WBRT) or stereotactic radiosurgery (SRS) are the main treatments for BM, and treatment selection is mainly based on the number of BMs $(15,16)$. BMs are often accompanied by peritumoral brain edema (PTBE). WBRT can cause or aggravate edema, leading to the symptom of intracranial hypertension (17). There is a synergistic effect between anti-angiogenic therapy and radiotherapy, as the VEGF signaling pathway is involved in the formation of PTBE in lung cancer patients with BMs (18). Anti-angiogenic therapy can normalize blood vessels and improve cell hypoxia, thus increasing the sensitivity of patients to radiotherapy (19-21). Thus, antiangiogenesis therapy combined with brain radiotherapy is the focus of much research, and its purpose is to improve the intracranial control rate and mitigate brain edema.
Previous studies have shown that the use of bevacizumab combined with radiotherapy in the treatment of BMs achieves satisfactory results and has good safety (22-24). Compared to bevacizumab, apatinib has a better application prospect because it is an oral dosage, which is easier to administer clinically, and it is also cheaper. So far, there is no definite evidence that apatinib can enter the bloodbrain-barrier, but a clinical study in small sample sizes have shown that apatinib can be sensitized to brain radiation (25). The highlight of our study is our radiotherapy modality (WBRT-SIB), which is a viable alternative to SRS for several reasons. First, many hospitals throughout the world do not have the proper equipment and technical capabilities to offer SRS, and this is especially true for rural facilities and/or those in developing nations. Second, the risk of radiation necrosis from SRS (especially for large $\mathrm{BM}$ ) is not trivial; the risk of radiation necrosis is quite low with a 15 -fraction SIB approach, and we have to date not observed a single case. Third, SRS is often not covered by insurance for numerous BM, and WBRT-SIB may offer an alternative path to dose-escalation of measurable BM that obviates insurance concerns. Until now, the efficacy of apatinib combined with WBRT-SIB for BMs has not been entirely clear. Thus, apatinib combined with WBRT-SIB was used to treat NSCLC patients with BMs at multiple centers. We retrospectively investigated the real-world efficacy and safety of this treatment model. We present the following article in accordance with the STROBE reporting checklist (available at https://jtd.amegroups.com/article/ view/10.21037/jtd-22-96/rc).

\section{Methods}

\section{Patients}

This retrospective study was jointly conducted by the Hubei Cancer Hospital, Renmin Hospital of Wuhan University, and Jianghan Oilfield Hospital. Eligibility criteria were patients 18-75 years old with measurable BM from histologically-confirmed NSCLC (including both newlydiagnosed and previously treated NSCLC) and expected survival time greater than 3 months. Patients were excluded from the study if they met any of the following exclusion criteria: had an uncontrolled or symptomatic systemic disease, such as active hepatitis B, AIDS, or Alzheimer's disease, had recurrent BM, and/or had previously received surgery or radiotherapy for BM (2 patients were excluded for recurrent $\mathrm{BM})$. 


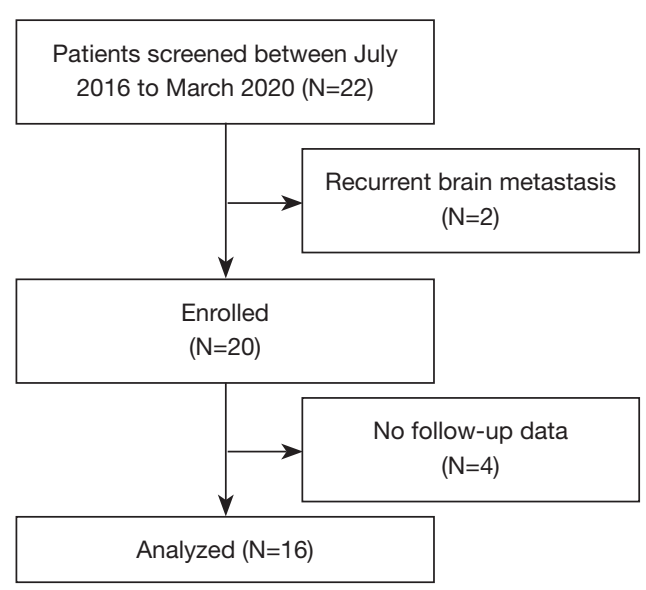

Figure 1 The trial profile of the screening process.

The patients' data, including data on their general condition, gene mutation status, intracranial tumor status, and adverse reactions, were retrospectively reviewed from electronic medical records. Patient survival was determined by electronic medical records or telephone follow-up calls (4 patients were excluded due to loss during the follow-up period).

The study was performed in accordance with the Declaration of Helsinki (as revised in 2013). The study was approved by the institutional review boards of the Hubei Cancer Hospital (No. LLHBCH2022YN-004), Renmin Hospital of Wuhan University (No. 2017K-C021), and Jianghan Oilfield Hospital. All the patients enrolled in this study signed the informed consent form. The patient selection procedure is displayed in Figure 1.

\section{Treatment model}

The specific implementation plan of apatinib combined with brain radiotherapy was as follows: 250 or $500 \mathrm{mg}$ of apatinib was administered orally 1 week before brain radiotherapy. The dose of apatinib was chosen according to the general condition of the patients. For patients with a Karnofsky performance score (KPS) of $80-90$ and those aged $<60$, $500 \mathrm{mg}$ of apatinib was administered orally, while the rest of the patients were administered $250 \mathrm{mg}$ of apatinib orally. If a patient had adverse reactions $>$ grade 2 , the dose of apatinib was reduced to $250 \mathrm{mg}$. For patients with epidermal growth factor receptor (EGFR) mutations, EGFR inhibitor drugs were administered at the same time as apatinib. The radiation dose for WBRT was 37.5 Gy/15 fractions
(F) with a simultaneous boost of 49.5-52.5 Gy/15 F. Apatinib was administered orally during and 1 week after radiotherapy. Intracranial progression-free survival (iPFS), OS, the intracranial disease control rate (iDCR), the intracranial overall response rate (iORR), and safety were calculated and observed.

\section{Patient evaluations}

Before starting treatment, patients received the relevant tests, including a complete blood count, liver and kidney function, blood pressure monitoring, routine urine tests, and brain MRI examinations. Each patient's KPS, neurological examination results, and physical examination results were also evaluated. After treatment and before the data block time, the patients were followed-up every 3 months until death or loss during follow-up. Brain MRI examinations were also conducted every 3 months during the follow-up period. Drug toxicity was graded according to the Common Terminology Criteria for Adverse Events V4.0 every week.

\section{Statistical analysis}

For the analysis of time to iPFS, the data for patients who were alive and had no intracranial disease progression regardless of the status of the extracranial lesions or who were lost to follow-up were censored at the time of the last tumor assessment. SPSS software (version 20.0) was used to conduct the statistical analysis of the data.

\section{Results}

\section{Patient's demographics}

From July 2016 to January 2020, 22 patients were treated under this model; however, after applying the patient selection procedure, only 16 patients who had been histologically confirmed NSCLC were enrolled in this retrospective study. As Table 1 shows, the most common pathological type was adenocarcinoma. Four patients had EGFR gene mutations (3 patients had harbor exon 19 deletions, and 1 patient had the exon 21 L858R mutation). There were 12 male and 4 female patients, and patients had a median age of 58 years (range, 33-74 years). Most patients had a KPS of $80-90$, and the majority $(62.5 \%)$ of patients had 1-3 BM. The median Diagnosis-Specific Graded Prognostic Assessment score was 2.5. 
Table 1 Clinical characteristics of the study population $(n=16)$

\begin{tabular}{lc}
\hline Characteristics & $\begin{array}{c}\text { Value or No. of } \\
\text { patients }\end{array}$
\end{tabular}

Age, years

Median 58

Range

33-74

Sex

Male

10

Female

6

62.5

No. of brain metastases

$0-3$

$4-10$

$>10$

10

4

2

Karnofsky performance score

100
90
80
70
60

Pathological pattern

Squamous

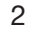

Adenocarcinoma

EGFR mutation status

With EGFR mutation

Without EGFR mutation

Smoking status

\begin{tabular}{lcc} 
Prior & 4 & 25.0 \\
Never & 5 & 31.25 \\
Current & 5 & 31.25 \\
Unknown & 2 & 12.5 \\
GPA & & \\
0.5 & 1 & 6.25 \\
1 & 1 & 6.25 \\
1.5 & 3 & 18.75 \\
2 & 3 & 18.75 \\
2.5 & 6 & 37.5 \\
3 & 2 & 12.5 \\
\hline EGFR epidermal growth factor receptor; GPA graded prognostic
\end{tabular}

EGFR, epidermal growth factor receptor; GPA, graded prognostic assessment.

\section{Toxicity}

All 16 patients in the study tolerated the treatment of apatinib combined with brain radiotherapy. Nine patients started taking $500 \mathrm{mg}$ of apatinib, but the dose of 2 patients was reduced to $250 \mathrm{mg}$ because of high blood pressure and oral mucositis and ulcers. The toxicity results are displayed in Table 2. The most common adverse reactions were hypertension and oral mucositis, both of which had an incidence of $18.75 \%$. Apatinib had little effect on bone marrow and liver function. Notably, there were no serious adverse reactions among patients taking EGFR inhibitors and apatinib at the same time. No grade 4-5 treatmentrelated toxicity was observed.

\section{Outcomes}

As of the most recent follow-up date (February 1, 2021), 7 patients were still alive. The median follow-up time was 16 months (range, 2-41 months). As Figure 2 and Table 3 shows, the median iPFS was 16.5 months [95\% confidence interval (CI): 15.1-37.4 months], and the median OS was 26 months (95\% CI: $17.0-54.0$ months). The iPFS times for 6 months, 1 year, and 2 years were $100 \%$, $68.8 \%$, and $18.8 \%$, respectively, while the OS times for 6 months, 1 year, and 2 years were $100 \%, 87.5 \%$, and $56.3 \%$, respectively.

According to the Response Evaluation Criteria in Solid Tumors (RECIST 1.1), as shown in Table 4, the intracranial complete response rate at 6 months after brain radiotherapy was $6.25 \%(\mathrm{n}=1)$, the intracranial partial response rate was $68.75 \%(\mathrm{n}=11)$, and the intracranial stable disease rate was $25 \%(n=4)$. Among the 16 patients, the iORR was $75 \%$ and the iDCR was $100 \%$. Additionally, the median brain EI score was initially 4.2, but after 3 months of brain radiotherapy, it was $1.9(\mathrm{P}=0.02)$. As shown in Figure 3, PTBE was significantly alleviated compared to that before treatment, and the brain EI score was significantly reduced.

\section{Discussion}

In this retrospective study, the median iPFS was 16.5 months, and the median OS was 26 months, both of which are longer than those of historical controls. Welsh conducted a study of erlotinib plus concurrent WBRT for patients with BMs, and found that the patients had a median central nervous system (CNS) progression-free survival time 
Table 2 Treatment-related toxicities

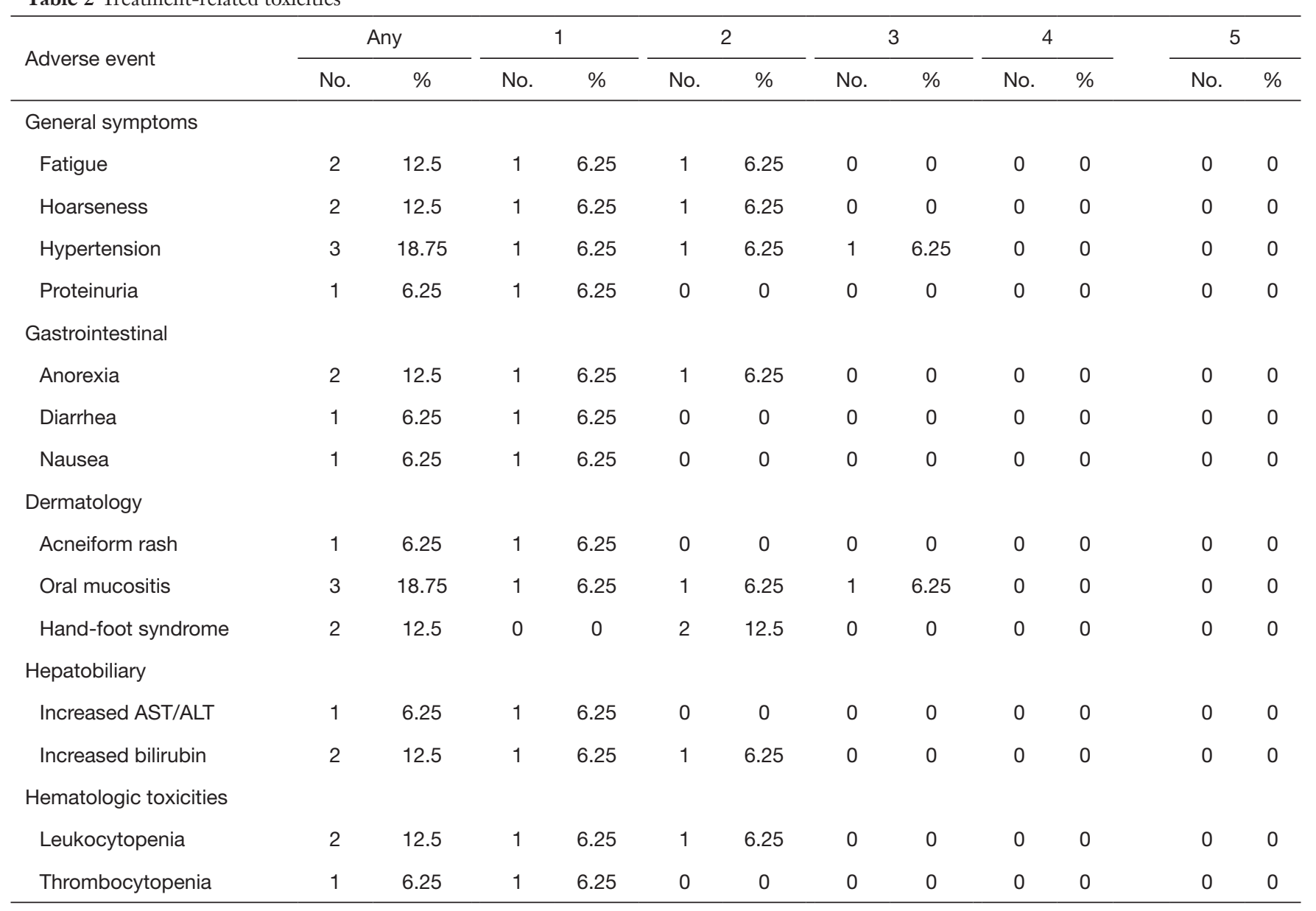

AST, aspartate aminotransferase; ALT, alanine amiotransferase.
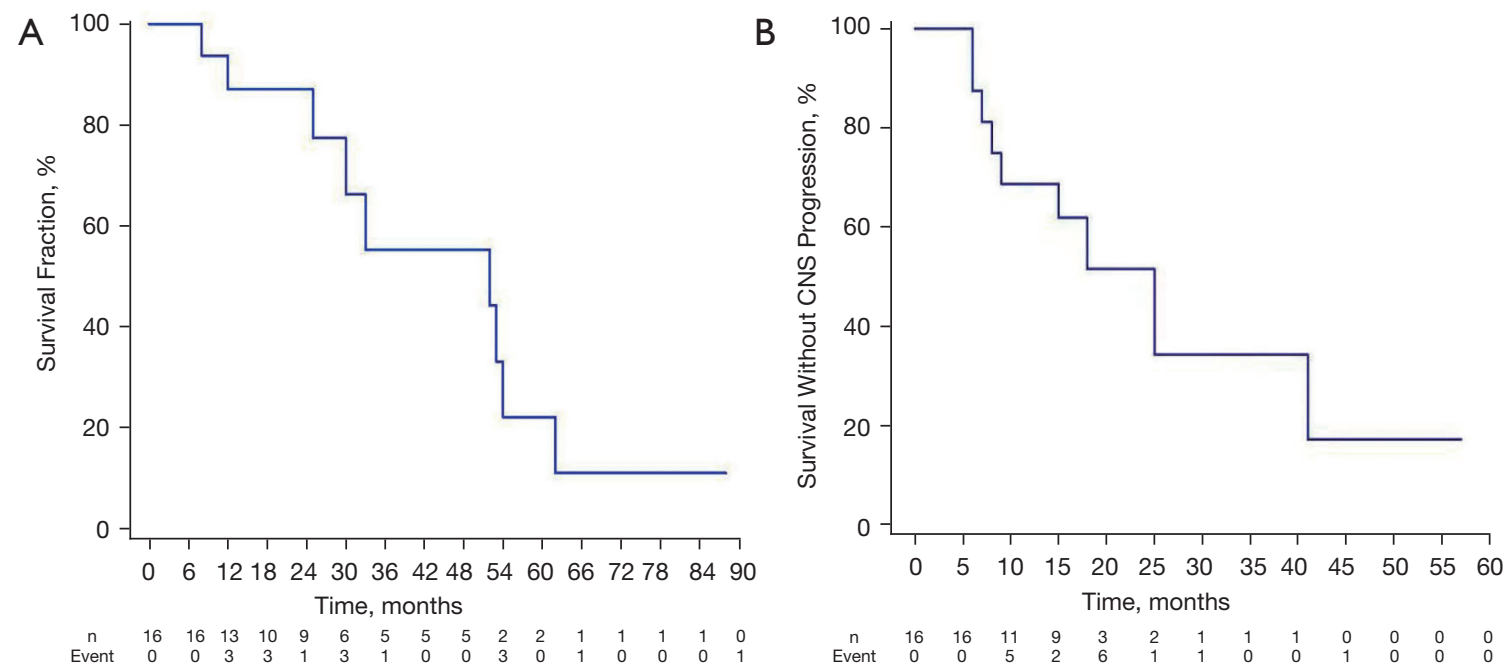

Figure 2 Kaplan-Meier curve for overall survival and intracranial progression free survival of the study population. (A) Overall survival of the study population. (B) intracranial progression free survival of the study population. 
Table 3 Outcomes of the trial population

\begin{tabular}{lcc}
\hline Outcome & Time (months) & (\%) \\
\hline Median overall survival time, months & 26 & \\
6-month rate & & 100 \\
1-year rate & 87.5 \\
2-year rate & 56.3 \\
Intracranial progression-free survival & 16.5 & \\
Median time, months & & \\
6-month rate & & 100 \\
1-year rate & 68.8 \\
2-year rate & 18.8 \\
\hline
\end{tabular}

of 8 months (26). Additionally, the BRAIN trial showed that for patients with the EGFR mutation in the WBRT group, the iPFS was only 4.8 months, while the median iPFS of patients in the icotinib group was 10.0 months (27). In our study, most patients had EGFR wild-type, but apatinib prolonged iPFS and even exceeded the iPFS of patients who received EGFR-TKI drugs combined with radiotherapy, which suggests that the use of apatinib combined with brain radiotherapy has strong potential to inhibit the progression of brain tumors. Based on the results of this retrospective study, we published a protocol of an open-label study of apatinib combined with brain radiotherapy in patients with driver-mutation negative NSCLC (28).
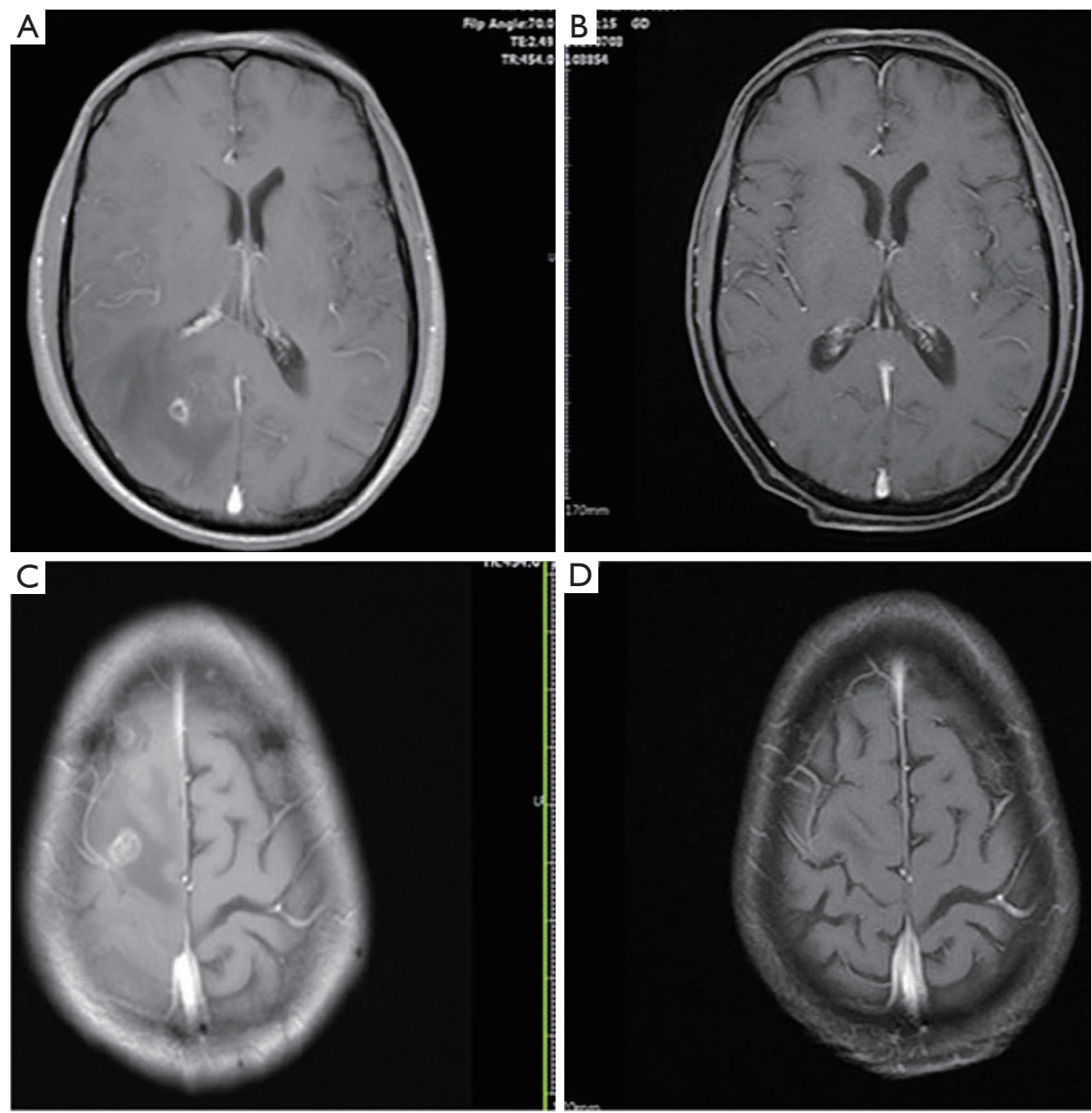

Figure 3 Brain metastases before and after the study regimen. (A) Pre-treatment right parieto-occipital lobe lesion with marked peritumoral edema. (B) Post-therapy showing an excellent response. (C) Multiple metastases with edema in the right frontal lobe prior to therapy. (D) Following therapy, an excellent response was observed. 
Table 4 BMs responses of the trial population

\begin{tabular}{lcc}
\hline BMs RECIST response & $\mathrm{n}$ & $\%$ \\
\hline CR & 1 & 6.25 \\
PR & 11 & 68.75 \\
SD & 4 & 25 \\
PD & 0 & 0 \\
\hline
\end{tabular}

BMs, brain metastases; RECIST, Response Evaluation Criteria in Solid Tumors; CR, complete response; PR, partial response; SD, stable disease; $\mathrm{PD}$, progressive disease.

Apatinib is a small-molecule antiangiogenic targeting agent that was independently developed in China. Apatinib selectively binds to VEGFR-2, and inhibits its function by inhibiting the formation of tumor blood vessels. Thus, apatinib effectively plays an anti-tumor role by inhibiting the proliferation, migration, and angiogenesis of vascular endothelial cells $(29,30)$. Additionally, PTBE in patients with BMs is closely associated with the VEGF signaling pathway. Thus, theoretically, inhibiting angiogenesis should reduce brain edema (31). Previous studies have shown that anti-angiogenesis drugs have a synergistic effect when combined with brain radiotherapy (20,22-24). Bevacizumab is the most studied drug in combination with brain radiotherapy. Compared to bevacizumab, apatinib is cheaper, can be taken orally, and is more convenient to use. In addition, patients in our study achieved a relatively long iPFS and OS compared to other similar clinical studies. Moreover, the brain EI decreased significantly after treatment, and the relief of brain symptoms before radiotherapy, such as nausea, vomiting and headache, was more obvious and rapid.

Equally important, the patients in our study tolerated the treatment well. The main adverse reactions were grades $1-2$, and the most common adverse events were oral mucositis, hypertension, hoarseness, and fatigue. The main reason that patients can tolerate apatinib well is because they do not take it for a very long time (usually only about 5 weeks).

In conclusion, apatinib combined with WBRT-SIB for BMs from NSCLC is safe and effective. Apatinib combined with brain radiotherapy effectively prolongs the iPFS, results in a better OS, and quickly reduces brain edema, thus largely reducing the symptoms of intracranial hypertension. This retrospective study identified a potential new therapeutic model of oral anti-angiogenic drugs combined with brain radiotherapy, which could be especially effective for patients whose have wild-type driver genes. However, because the sample size of this retrospective study was small, multicenter, large sample size studies need to be conducted to verify our findings in the future.

\section{Acknowledgments}

Funding: This work was supported by the National Natural Science Foundation of China (No. 81903137), the Chinese Society of Clinical Oncology Foundation (No. Y-HR2016-44) and Hubei Provincial Health Department Foundation (No. ZY2021M008).

\section{Footnote}

Reporting Checklist: The authors have completed the STROBE reporting checklist. Available at https://jtd. amegroups.com/article/view/10.21037/jtd-22-96/rc

Data Sharing Statement: Available at https://jtd.amegroups. com/article/view/10.21037/jtd-22-96/dss

Conflicts of Interest: All authors have completed the ICMJE uniform disclosure form (available at https://jtd.amegroups. com/article/view/10.21037/jtd-22-96/coif). The authors have no conflicts of interest to declare.

Ethical Statement: The authors are accountable for all aspects of the work in ensuring that questions related to the accuracy or integrity of any part of the work are appropriately investigated and resolved. The study was approved by the institutional review boards of the Hubei Cancer Hospital (No. LLHBCH2022YN-004), Renmin Hospital of Wuhan University (No. 2017K-C021), and Jianghan Oilfield Hospital. All the patients enrolled in this study signed an informed consent form. The study was performed in accordance with the Declaration of Helsinki (as revised in 2013).

Open Access Statement: This is an Open Access article distributed in accordance with the Creative Commons Attribution-NonCommercial-NoDerivs 4.0 International License (CC BY-NC-ND 4.0), which permits the noncommercial replication and distribution of the article with the strict proviso that no changes or edits are made and the original work is properly cited (including links to both the formal publication through the relevant DOI and the license). See: https://creativecommons.org/licenses/by-nc-nd/4.0/. 


\section{References}

1. Villano JL, Durbin EB, Normandeau C, et al. Incidence of brain metastasis at initial presentation of lung cancer. Neuro Oncol 2015;17:122-8.

2. Gavrilovic IT, Posner JB. Brain metastases: epidemiology and pathophysiology. J Neurooncol 2005;75:5-14.

3. Eichler AF, Chung E, Kodack DP, et al. The biology of brain metastases-translation to new therapies. Nat Rev Clin Oncol 2011;8:344-56.

4. Kamar FG, Posner JB. Brain metastases. Semin Neurol 2010;30:217-35.

5. Posner JB. Management of brain metastases. Rev Neurol (Paris) 1992;148:477-87.

6. Sperduto PW, Kased N, Roberge D, et al. Summary report on the graded prognostic assessment: an accurate and facile diagnosis-specific tool to estimate survival for patients with brain metastases. J Clin Oncol 2012;30:419-25.

7. Folkman J. Antiangiogenesis in cancer therapy--endostatin and its mechanisms of action. Exp Cell Res 2006;312:594-607.

8. Pradeep CR, Sunila ES, Kuttan G. Expression of vascular endothelial growth factor (VEGF) and VEGF receptors in tumor angiogenesis and malignancies. Integr Cancer Ther 2005;4:315-21.

9. Sandler A, Gray R, Perry MC, et al. Paclitaxel-carboplatin alone or with bevacizumab for non-small-cell lung cancer. N Engl J Med 2006;355:2542-50.

10. Han B, Li K, Wang Q, Zhang L, et al. Effect of Anlotinib as a Third-Line or Further Treatment on Overall Survival of Patients With Advanced Non-Small Cell Lung Cancer: The ALTER 0303 Phase 3 Randomized Clinical Trial. JAMA Oncol 2018;4:1569-75.

11. Cheng Y, Wang Q, Li K, et al. Anlotinib vs placebo as third- or further-line treatment for patients with small cell lung cancer: a randomised, double-blind, placebocontrolled Phase 2 study. Br J Cancer 2021;125:366-71.

12. Li J, Qin S, Xu J, et al. Apatinib for chemotherapyrefractory advanced metastatic gastric cancer: results from a randomized, placebo-controlled, parallel-arm, phase II trial. J Clin Oncol 2013;31:3219-25.

13. Li J, Qin S, Xu J, et al. Randomized, Double-Blind, Placebo-Controlled Phase III Trial of Apatinib in Patients With Chemotherapy-Refractory Advanced or Metastatic Adenocarcinoma of the Stomach or Gastroesophageal Junction. J Clin Oncol 2016;34:1448-54.

14. Scott LJ. Apatinib: A Review in Advanced Gastric Cancer and Other Advanced Cancers. Drugs 2018;78:747-58.

15. Knisely JP, Berkey B, Chakravarti A, et al. A phase III study of conventional radiation therapy plus thalidomide versus conventional radiation therapy for multiple brain metastases (RTOG 0118). Int J Radiat Oncol Biol Phys 2008;71:79-86.

16. McHaffie DR, Chabot P, Dagnault A, et al. Safety and feasibility of motexafin gadolinium administration with whole brain radiation therapy and stereotactic radiosurgery boost in the treatment of $\leq 6$ brain metastases: a multiinstitutional phase II trial. J Neurooncol 2011;105:301-8.

17. Venur VA, Funchain P, Kotecha R, et al. Changing Treatment Paradigms for Brain Metastases From Melanoma-Part 1: Diagnosis, Prognosis, Symptom Control, and Local Treatment. Oncology (Williston Park) 2017;31:602-6.

18. Jun KH, Lee JE, Kim SH, et al. Clinico pathological significance of $\mathrm{N}$-cadherin and VEGF in advanced gastric cancer brain metastasis and the effects of metformin in preclinical models. Oncol Rep 2015;34:2047-53.

19. Chen G, Liu XY, Wang Z, et al. Vascular endothelial growth factor $\mathrm{C}$ : the predicator of early recurrence in patients with N2 non-small-cell lung cancer. Eur J Cardiothorac Surg 2010;37:546-51.

20. Strugar J, Rothbart D, Harrington W, et al. Vascular permeability factor in brain metastases: correlation with vasogenic brain edema and tumor angiogenesis. J Neurosurg1994;81:560-6.

21. Sofia Vala I, Martins LR, Imaizumi N, et al. Low doses of ionizing radiation promote tumor growth and metastasis by enhancing angiogenesis. PLoS One 2010;5:e11222.

22. Wang Y, Wang E, Pan L, et al. A new strategy of CyberKnife treatment system based radiosurgery followed by early use of adjuvant bevacizumab treatment for brain metastasis with extensive cerebral edema. J Neurooncol 2014;119:369-76.

23. Yomo S, Hayashi M. Salvage stereotactic radiosurgery with adjuvant use of bevacizumab for heavily treated recurrent brain metastases: a preliminary report. J Neurooncol 2016;127:119-26.

24. Lévy C, Allouache D, Lacroix J, et al. REBECA: a phase I study of bevacizumab and whole-brain radiation therapy for the treatment of brain metastasis from solid tumours. Ann Oncol 2014;25:2351-6.

25. Welsh JW, Komaki R, Amini A, et al. Phase II trial of erlotinib plus concurrent whole-brain radiation therapy for patients with brain metastases from non-small-cell lung cancer. J Clin Oncol 2013;31:895-902.

26. Ren Y, Wang SB, Zhou L, et al. Continuous LowDose Apatinib Combined With WBRT Significantly 
Reduces Peritumoral Edema and Enhances the Efficacy of Symptomatic Multiple Brain Metastases in NSCLC. Technol Cancer Res Treat 2021;20:15330338211011968.

27. Yang JJ, Zhou C, Huang Y, et al. Icotinib versus wholebrain irradiation in patients with EGFR-mutant non-smallcell lung cancer and multiple brain metastases (BRAIN): a multicentre, phase 3, open-label, parallel, randomised controlled trial. Lancet Respir Med 2017;5:707-16.

28. Ma J, Pi G, Bi J, et al. Concurrent Apatinib and Brain Radiotherapy in Patients With Brain Metastases From Driver Mutation-negative Non-small-cell Lung Cancer: Study Protocol for an Open-label Randomized Controlled

Cite this article as: Ma J, Bi J, Tuo X, Pi G, Li Y, Li Y, Zeng F, Gong H, Hu D, Han G. Efficacy and safety of apatinib combined with whole-brain radiation therapy with a simultaneous integrated boost for brain metastases from nonsmall cell lung cancer: a multicenter retrospective study. J Thorac Dis 2022;14(2):455-463. doi: 10.21037/jtd-22-96
Trial. Clin Lung Cancer 2021;22:e211-4.

29. Wang C, Jiang M, Hou H, et al. Apatinib suppresses cell growth and metastasis and promotes antitumor activity of temozolomide in glioma. Oncol Lett 2018;16:5607-14.

30. Fang Z, Feng S, Zhang J, et al. Combined treatment of apatinib with docetaxel in non-small-cell lung cancer mice and its material basis of pharmacokinetics. J Clin Oncol 2017;35:e14069.

31. Wang W, DA R, Wang M, et al. Expression of brainspecific angiogenesis inhibitor 1 is inversely correlated with pathological grade, angiogenesis and peritumoral brain edema in human astrocytomas. Oncol Lett 2013;5:1513-8. 